\title{
Analysis of Different Path Loss Models in Urban Suburban and Rural Environment
}

\author{
Sreevardhan Cheerla, K. Sindhuja, Ch. Indra Kiran, D. Ajaya Venkatesh \\ Department of Electronics and Communication Engineering, Koneru Lakshmaiah Education Foundation, Green \\ Fields, Vaddeswaram, Guntur (Dist), AP, India-522502 \\ sreevardhancheerla@kluniversity.in
}

\begin{abstract}
Radio proliferation with the structure, arrangement and the executives of a remote system are the fundamentals for developing innovations. It is extremely site-specific and depending on the area, operational rate, moving terminal speed, device sources and other variable element, can differ considerably. For the prevision of signal coverage, it is important to properly characterize the radio channel using key parameters and a mathematical model. Path loss models are analyzed and compared with their parameters for macro cells like Hata, COST231 and Ericsson models. If there is a long-distance communication between transmitter and receiver with very high frequencies the signals are affected by various path loss effects in the environment. So, the main objective of this paper is to compare the various path loss models with the measured data and identifying the error and concluding which model is the most preferable with respective to measured data. This paper focuses on path loss models at different environments like Urban,Sub-Urban and Rural areas.
\end{abstract}

Key words: Macro cells, Path loss models, COST 231, hata.

\section{INTRODUCTION}

The loss which occurs in between transmitter and receiver is called as propagation path loss. It is essential for the path loss to be predicted because it depends on different factors like Connectivity, Reliability and Latency factors[1,2]. If the transmitted signal is received at the receiver end with less noise, then this terminology is treated as Reliability. Connectivity deals with the signal and network device connections with in the medium. Latency speaks about the delay before transferring the data.

Depending upon the losses the path loss is categorized in to three categories. Empirical, Deterministic and statistical models $[3,4]$. Here Empirical path loss model is commonly used for predicting path loss in mobile wireless communication. COST231, Hata and Ericsson are the empirical models [2,4,11]. The path loss depends upon transmitter and receiver coefficients like transmitting antenna height, receiving antenna height, width, frequency, and distance [2]. The various methods are implemented to determine to predict the path loss. Different path loss models are presented in a graph along with the measured data. The root mean square error (RMSE) has been used as standard statistical metric to measure model performance in air quality, and also climate research studies. The testing criteria of this paper are to compare measured data with different Path loss models. The main criteria are to measure and find out the error and predicting which will be the most appropriate model. The next few sections of this paper consist of different Path loss models, detecting the error using RMSE and finding out the results and discussion and finally the conclusion.

\section{PATH LOSS}

Path loss defines decreasing the amount of power with respect to volume parameter in an electromagnetic wave where the signal is passing through free medium $[3,5]$. For example, if there is a transmitter antenna with transmits power on one side and if we transmit it into isotropic source the signal is going to be radiated equally in all the directions. On the other side if the consideration is on receiving antenna with received power, the power at which the transmitter produces will definitely be greater than that the power at the receiver. This is because the power which is transmitted will be transmitting in all the directions and only some power is received at the receiver end. In an isotropic antenna the formula for the path loss is described in equation 1 .

$$
\text { Pathloss }=20 \log \left(\frac{4 \pi d}{\lambda}\right) \rightarrow 1
$$

Where,

$\lambda$ value is followed by signal wavelength $\mathrm{d}$ value refers to the distance between antennas

Path loss is used to describe the attenuation of an electromagnetic wave and it propagates through air space [6]. Path loss prediction experimental analysis are done in three types of areas they are referred as urban, rural and suburban[2,3,5]. In rural environment there will be Line of sight so the signal received will be more accurate when compared to suburban and urban areas in urban areas fading occurs because height of mobile antenna is less than surrounding structures [4]. 


\section{VARIOUS PATH LOSS MODELS}

\subsection{Ericsson Model}

Ericsson is one of the types of Terrain model and is used for radio frequency propagation. This software is used by the network engineers in Ericsson company, so this model is called Ericsson model [4]. The ericsson path loss calculation is done by the equation 2 and 3 [5]:

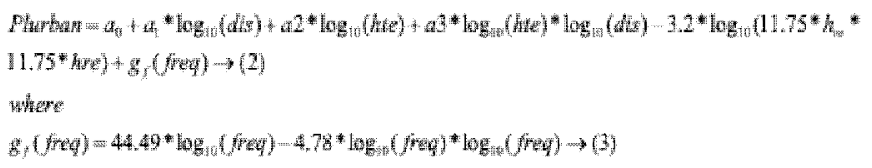

Ericsson parameters are illustrated in table 1.

Table 1: Ericsson parameters

\begin{tabular}{l|llll}
\hline $\begin{array}{l}\text { Path loss } \\
\text { environmental } \\
\text { conditions }\end{array}$ & A0 & A1 & A2 & A3 \\
\hline Suburban & 43.200 & 68.930 & 12.00 & 0.100 \\
Urban & 36.200 & 30.200 & 12.00 & 0.100 \\
Rural & 45.950 & 100.60 & 12.00 & 0.100 \\
\hline
\end{tabular}

Different Path loss environmental conditions and their Ericsson parameters at Suburban, Urban, and Rural areas.

\subsection{Hata Model}

The Hata model is used for predicting path loss of cellular transmission in exterior environments [2]. It is associated with Empirical formulation based on data form the Okumura model, which is referred as Okumura-Hata model. There are plenty of specific models for special uses. Its frequency range $150 \mathrm{MHz}$ to $1500 \mathrm{MHz}[2,7]$. Propagation losses will increase with frequency and predicting the behavior of cellular communication in designed-up areas.

Hata model is suited for both point to point and broadcast communication. It can cover base station height range from 30 $\mathrm{mts}$ to $200 \mathrm{mts}$ and mobile base station height range from $1 \mathrm{mt}$ to $10 \mathrm{mts}[8,11]$. In open area the territory having open space and no tall tree or structures ought to be in way, and a plot of land ought to be cleared at any rate $300-400 \mathrm{~m}$ for example ranch land, rice fields, open fields. In sub-urban area the territory having Town or interstate dissipated with trees and houses, there is a probability of certain hindrances close to the portable pinnacle anyway it ought not be blocked. Path loss is regularly utilized in both remote and signal communication interchanges. There such huge numbers of elements like free space misfortune, Refraction Diffraction, Reflection, Gap will influence the path loss[1,2].
According to the prediction models use building highs, street width, street orientation etc. in the short-distance path loss estimate. Such designs are used in structures of micro-cells. If the width of the cells is relatively small (range 10 to $100 \mathrm{~m}$ ), the ray-tracking system is used for deterministic models. It is therefore essential for the design of a mobile system to be selected in a given environment as a path-loss model[9]. Macrocells are described with several experimental route loss models. Path loss is regularly utilized in both remote and signal communication interchanges.

\subsubsection{Hata Model for Urban}

Hata model is the most overall used radio repeat spread model for foreseeing the lead of cell transmissions in organized up zones. This specific interpretation of the Hata model is relevant to the radio multiplication among urban districts. This model doesn't offer consideration to the total move of frequencies secured by the Okumura model. Hata model range is greater compared to Okumura. The hata path loss calculation is done by the equation 4, 5 and 6 .

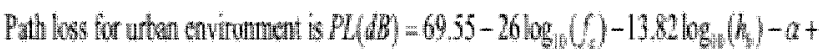

$$
\begin{aligned}
& \left(44.9-6.55 \log _{0}(k)(D) \rightarrow(4)\right.
\end{aligned}
$$

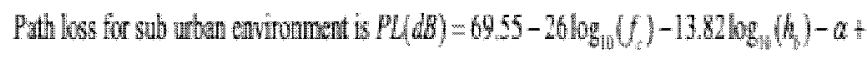

$$
\begin{aligned}
& \left(44.9-6.55 \log _{3}(h)(D)-\left(24 \log _{0}\left(\frac{f_{0}}{28}\right)^{2}+5.4\right)+(9)\right. \\
& \text { Path loss for rual envitument is } P(d B)=69.55-26 \log _{3}(0)-13.82 \log _{3}\left(h_{4}\right)-a-
\end{aligned}
$$

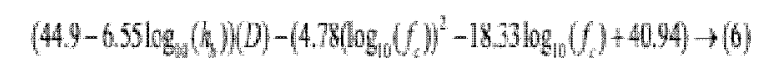

$$
\begin{aligned}
& a=3.2\left(\log _{\mathrm{g}}\left(11,75 \mathrm{~h}_{\mathrm{m}}\right)\right)^{2}-4.97 \cdots f_{\mathrm{r}}>300 \mathrm{MH} \\
& \tilde{a}=8.29\left(\log _{0}\left(1.54 i_{m}\right)\right)^{2}-1.1 \cdots f<300 \mathrm{MHz}
\end{aligned}
$$

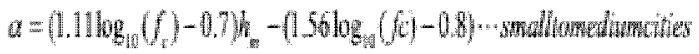

Where,

$\mathrm{hm}$ is the mobile station height

$\mathrm{f}$ is the frequency in $\mathrm{Hz}$

\subsection{COST-231 Model}

The COST-231 model is used for predicting path loss in wireless communication system[2,6]. This model is utilized distinctly specifically bandwidth from $500 \mathrm{MHz}$ to $2.1 \mathrm{GHz}$ $(2000 \mathrm{MHz})$ on the grounds that is the recurrence of the versatile[2,11]. The condition is the $\mathrm{cm}$ esteem in the above condition will be 0 (zero) for the littler urban communities and the $\mathrm{cm}$ worth will be $3 \mathrm{~dB}$ for bigger urban communities. At the point when he is the CPE (Client Premises Gear) radio wire tallness above the ground. To assess the materialness of the model for the COST- $231 \mathrm{GHz}$ band, the model expectations depend on the estimations from three conditions in particular urban, rural and the country. The path loss calculation is done by the equation 7 and 8 [5]: 
Pathloss $=F+B \log R-\alpha+G \rightarrow$ (7)

where

$F=46.3+33.9 \log f_{c}-13.82 \log h_{b} \rightarrow(8)$

$G=\left\{\begin{array}{cc}0 d B \quad \text { for suburban and medium sized cities } \\ 3 d B \text { for Metropolitan cities }\end{array}\right\}$

\subsection{Root Mean Square Error}

Root Mean Square error is the standard deviation of prediction errors. RMSE tells you how the concentrated data around the line fits the best. It is commonly used in forecasting and regression analysis to verify experimental results. A better insight can be obtained by computing the root mean square error (RMSE) associated with each model using the following equation $9[6]$ :

$$
R M S E=\sqrt{\sum_{i=1}^{N} \frac{\left(P L_{m}-P L\right)^{2}}{N}} \rightarrow(9)
$$

Where,

$\mathrm{PL}_{\mathrm{m}}$ is the measured path loss in $\mathrm{db}$

PL is the predicted path loss in $\mathrm{dB}$

$\mathrm{N}$ is the number of measured data Points.

\section{METHODOLOGY}

A signal with $1800 \mathrm{MHz}$ frequency is used in order to compare the data in India at urban and sub-urban environments which are two different locations are Base Station 1(BTS1) and Base Station 2(BTS2). While choosing a best suitable model, one of the different types of path loss models is selected by comparing with one another. Theoretically it is showing that pathloss is positive and signal strength is negative. With the help of information, the error values are calculated in different pathloss models. The path loss depends upon transmitter and receiver coefficients, which are width frequency and distance between each other, which then followed by path loss node selection for users is executed. Then Root Mean Square Error(RMSE) values are calculated to know the performance of each model. The one which is having lower RMSE value is the best model compared to measured path loss.

\section{RESULTS AND DISCUSSION}

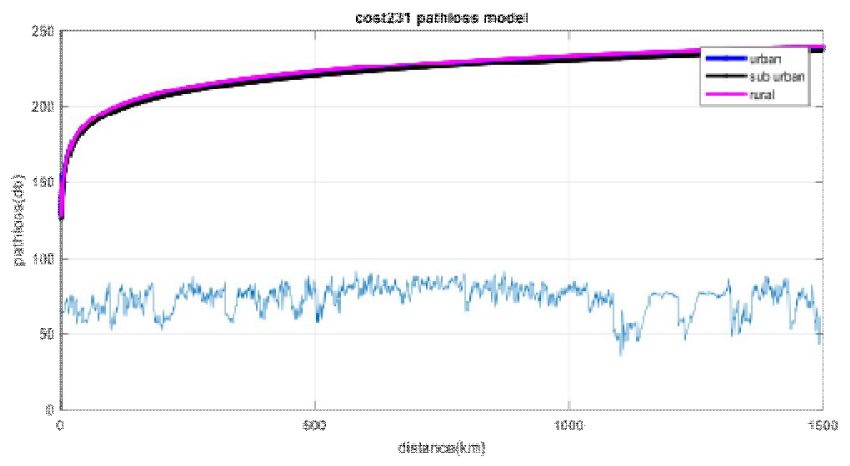

Figure 1: Comparison of measured data with path loss models in urban environment.

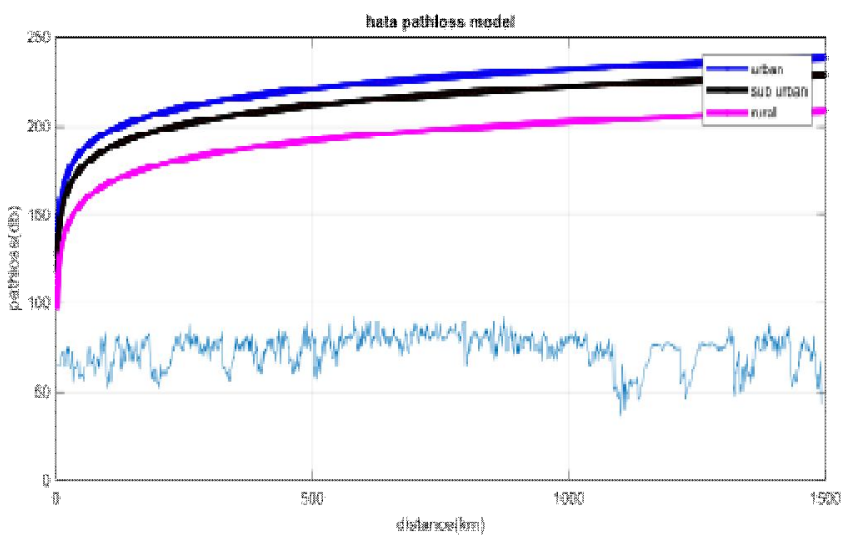

Figure 2: Comparison of measured data with path loss models in sub-urban environment.

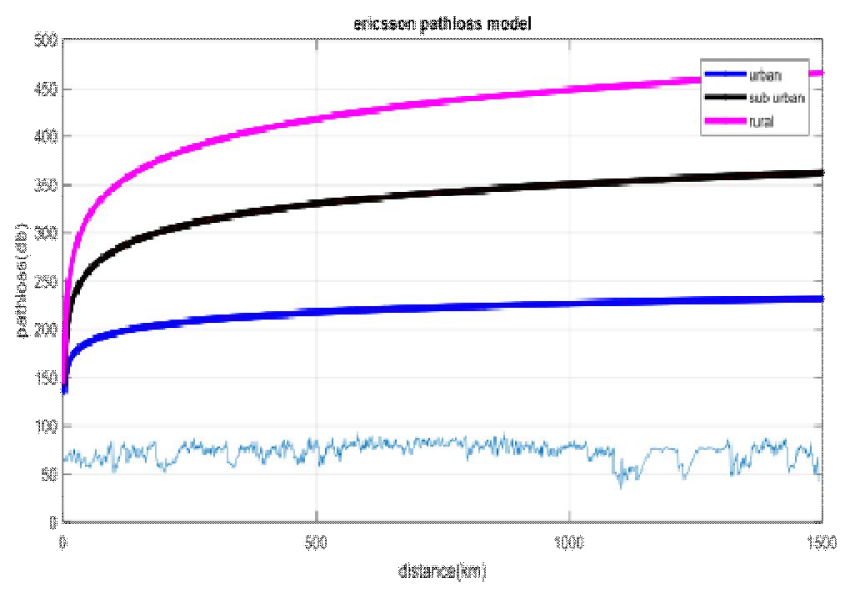

Figure 3: Comparison of measured data with path loss models in rural environment.

Figure 1 illustrates the path loss values at urban environment are stimulated using different path loss models. Hata model with blue, COST-231 with black, Ericsson with pink, measured data is the ECG signal is represented respectively. Here the measured data is compared with different path loss models to choose the appropriate path model for prediction. The path loss variation between hata and cost-231 models areless and both are similar. Figure 2 illustrates path loss values at sub-urban environment are stimulated using different path loss models. The path loss variation between models are increased while comparing to figure1. Here the measured data is compared with different path loss models at sub-urban areas. Figure 3 illustrates the path loss values at Rural areas are stimulated using different path loss models. Here the measured data is compared with different path loss models at rural areas. The path loss variations between models are increased by comparing with figure 1 and figure 2 . 


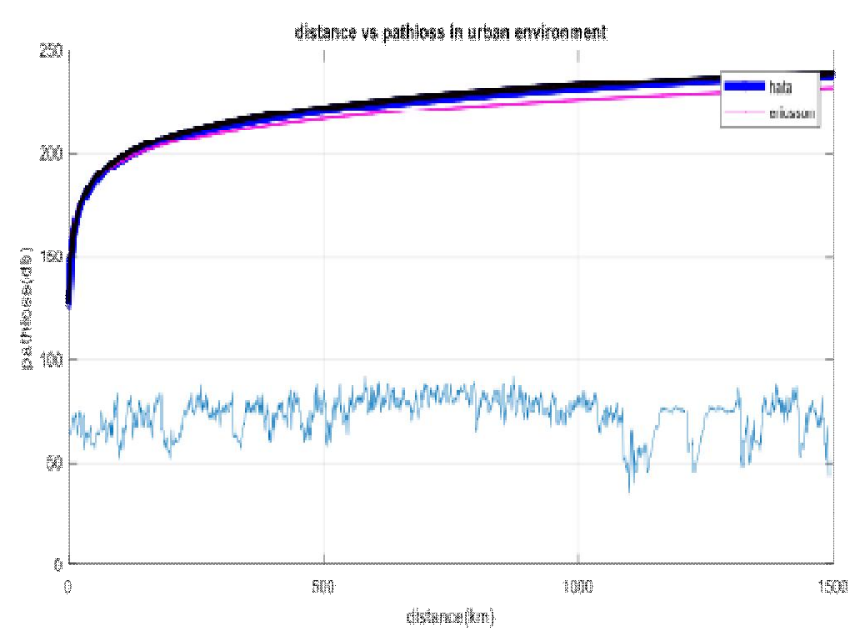

Figure 4: Comparison of measured data with all the environments in hata model.

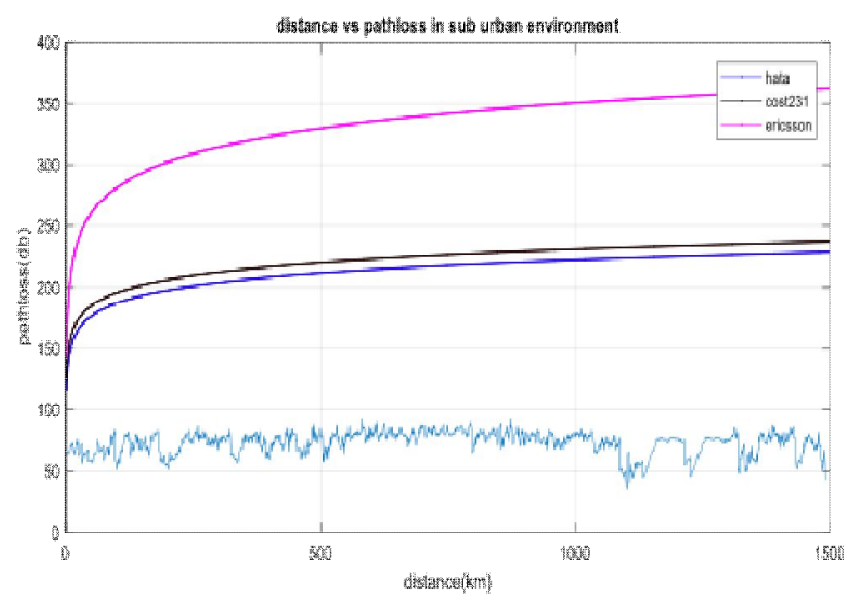

Figure 5: Comparison of measured data with all the environments in cost 231 model.

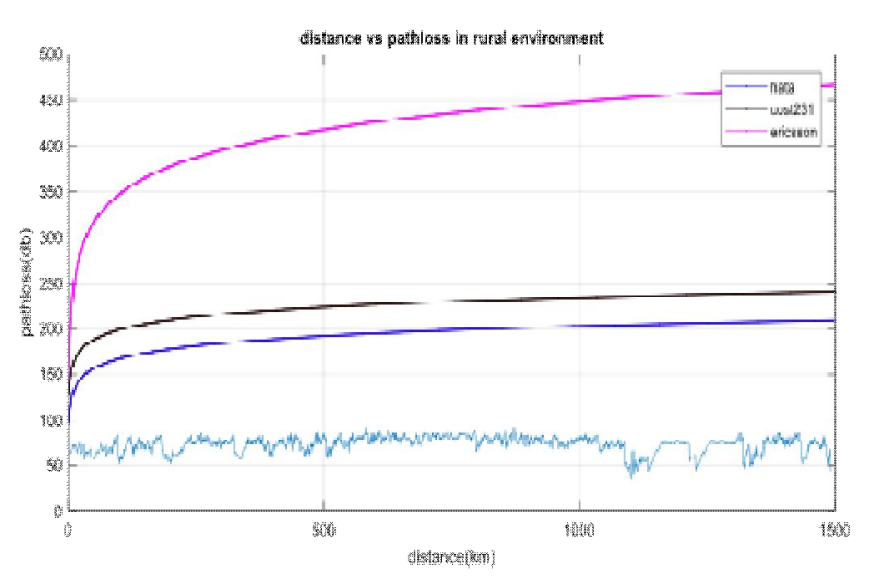

Figure 6: Comparison of measured data with all the environments in Ericsson model.
Figure 4 illustrates the comparison of measured data at different areas with hata path loss model. In these figures urban environment with blue, sub-urban environment with black, rural environment with pink, and measured path loss with ECG signal. While distance increases path $\operatorname{loss}(\mathrm{db})$ also increases. The path loss variation between all environments is high and increasing accurately. Figure 5 illustrates comparison of measured data at different areas with cost-231 path loss model. Comparing to figure 4 the path loss variation between all environments is less. While distance is increasing the path $\operatorname{loss}(\mathrm{db})$ is also increasing most accurately. Figure 6 illustrates comparison of measured data at different areas with ericsson pathloss model. While comparing with figure 5 and figure 6 measured data is less than urban and sub-urban environments. We observe that COST-231 is the most appropriate path loss model. RMSE values are given by Table 2 and 3.

Table 2: RMSE values based on environment of base station 1

\begin{tabular}{l|lcl}
\hline Environment & COST & HATA & ERICSSON \\
& 231 & & \\
\hline Urban & 0.1511 & 0.3973 & 0.1667 \\
Sub-Urban & 0.6263 & 2.5337 & 13.2634 \\
Rural & 0.0445 & 6.9720 & 23.0917 \\
\hline
\end{tabular}

The RMSE values for different pathloss models at urban, Sub-urban and Rural areas (BTS1).

Table 3: RMSE values based on environment of base station 2

\begin{tabular}{l|lcl}
\hline Environment & COST & HATA & ERICSSON \\
& 231 & & \\
\hline Urban & 0.1511 & 0.3973 & 0.1667 \\
Sub-Urban & 0.6263 & 2.5337 & 13.2634 \\
$\quad$ Rural & 0.0445 & 6.9720 & 23.0917 \\
\hline
\end{tabular}

The RMSE values for different path loss models at Urban,Sub-Urban and Rural areas(BTS 2).

Table2 and Table3 illustrates the RMSE values based on all environments of base station( $1 \& 2)$. This helps to know RMSE values of path loss for better understanding of the performance. In urban environment cost-231 model it was 0.1511 , and hata model it was 0.3973 , and ericsson model it was 0.1667 . In sub-urban environment the RMSE values are increased as cost-231 model it was 0.6263 , and hata model it was 2.5337, and ericsson model it was 13.2634. In rural environment the RMSE values are as cost-231 model it was 0.0445 , and hata model it was 6.9720 , and ericsson model it was 23.0917 . 


\section{CONCLUSION}

For Large scale cells in it is found that different regions have, different pattern of loss of tracks were used and compared. The MATLAB simulation shows that, with the Okumura-Hata model, the least track loss in urban areas and the maximum signal received at a given distance are generated. Likewise, a lack of control was explored at some suburban and rural areas using the Okumura-Hata system. The signal quality got is the farthest point. They assume therefore that the Okumura-Hata system is appropriate for both metropolitan, sub-urban and rural areas and RMSE values of COST PSO is lower when compared to other algorithms, this shows it has higher performance.

\section{REFERENCES}

1. Verma, R. and Saini, G., 2016, March. Statistical tuning of Cost-231 Hata model at $1.8 \mathrm{GHz}$ over dense urban areas of Ghaziabad. In 2016 3rd International Conference on Computing for Sustainable Global Development (INDIACom)(pp. 1220-1225). IEEE.

2. Emeruwa, C. and Iwuji, P.C., 2018. Determination Of A Pathloss Model For Long Term Evolution (Lte) In Yenagoa. The International Journal of Engineering and Science (IJES), pp.38-44.

3. Zreikat, A. and Dordevic, M., 2017. Performance analysis of path loss prediction models in wireless mobile networks in different propagation environments. In Proceedings of the 3Rd World Congress on Electrical Engineering and Computer Systems and Science (EECSS'17) Rome, Italy-June 4-6. https://doi.org/10.11159/vmw17.103

4. Bola, G.S. and Saini, G.S., 2013. Path loss measurement and estimation using different empirical models for WiMAX in urban area. International Journal of Scientific \& Engineering Research, 4(5), pp.1421-1428.

5. Eichie, J.O., Oyedum, O.D., Ajewole, M.O. and Aibinu, A.M., 2017. Comparative analysis of basic models and artificial neural network based model for path loss prediction. Progress In Electromagnetics Research, 61, pp.133-146.

https://doi.org/10.2528/PIERM17060601

6. Chebil, J., Lwas, A.K., Islam, M.R. and Zyoud, A., 2011. Investigation of path loss models for mobile communications in Malaysia. Australian Journal of Basic and Applied Sciences, 5(6), pp.365-371.

7. Nadir, Z. and Ahmad, M.I., 2010, March. Pathloss determination using Okumura-Hata model and cubic regression for missing data for Oman. In Proceedings of the International MultiConference of Engineering and Computer scientist (Vol. 2).

8. Hata, M., 1980. Empirical formula for propagation loss in land mobile radio services. IEEE transactions on Vehicular Technology, 29(3), pp.317-325.

https://doi.org/10.1109/T-VT.1980.23859
9. Sharma, P.K. and Singh, R.K., 2010. Comparative analysis of propagation path loss models with field measured data. International Journal of Engineering Science and Technology, 2(6), pp.2008-2013.

10. Egli, J.J., 1957. Radio propagation above $40 \mathrm{MC}$ over irregular terrain. Proceedings of the IRE, 45(10), pp.1383-1391.

11. Nwalozie, G.C., Ufoaroh, S.U., Ezeagwu, C.O. and Ejiofor, A.C., 2014. Path loss Prediction for GSM Mobile Networks for Urban Region of Aba, South-Eastern Nigeria. International Journal of Computer Science and Mobile Computing, 3(2), pp.83-90.

12. D Venkata Ratnam, Rakesh Chowdary Gutta, M. Ravi Kumar, L. Eswara Rao, M. V. Siva Surya Reddy, P. Sai Pavan, Chella Santhosh, Effect of High-K Dielectric materials on Mobility of Electrons, International Journal of Emerging Trends in Engineering Research, 2020, Vol. 8, Issue:2, pp. 314-316. https://doi.org/10.30534/ijeter/2020/12822020

13. M.Sridhar, M. Ravi Kumar, J.Chandana, S.V.Pranitha, K.Y.Sahithi, Chella Santhosh, Detection of Various Adulterants Concentration levels in Gasoline Using NI-LABVIEW, International Journal of Emerging Trends in Engineering Research, 2020, Vol. 8, Issue:4, pp. 1247-1250.

https://doi.org/10.30534/ijeter/2020/49842020 